OITS 531

FIAN TD-27

December 1993

\title{
QUARK AND GLUON JETS IN QCD: FACTORIAL AND CUMULANT MOMENTS
}

\author{
I.M.Dremin ${ }^{1}$ and R.C. $\mathrm{Hwa}^{2}$ \\ Institute of Theoretical Science, University of Oregon, \\ Eugene, Oregon 97403, USA
}

\begin{abstract}
Parton correlations in quark and gluon jets are studied by calculating moments of their multiplicity distributions in fixed coupling QCD. Exact solution of the problem for moments of any rank has been found. The ratio of cumulant to factorial moments reveals an oscillatory structure which is very sensitive to tiny details of the distributions.
\end{abstract}

PQด0อ219?

\footnotetext{
${ }^{1}$ Permanent address: P.N.Lebedev Physical Institute, Leninsky prospect,53, Moscow 117924, Russia. E.mail address: dremin@td.fian.free.net

${ }^{2}$ E.mail address: hwa@oregon.uoregon.edu
} 



\section{Introduction}

Among the various ways of testing perturbative QCD the study of the properties of multiparticle production in quark and gluon jets is both important and relatively straightforward, theoretically and experimentally. At this point some global properties predicted by pQCD are still not verified by data to high enough accuracy. In multiparticle production the primary observable is the multiplicity distribution $P_{n}$. The first moment of $P_{n}$ is the average multiplicity $\langle n\rangle$, which has attracted considerable attention in QCD [1]. We have calculated it recently for quark and gluon jets by solving exactly the fixed coupling QCD equations [2]. We now investigate the higher moments of $P_{n}$ so as to learn about the fluctuations from $\langle n\rangle$, and consequently also about the multiparticle correlations.

The starting point of this line of investigation is the differential-integral equations of the generating functions, as in [1] - [6]. The various efforts to determine the measurable implications of those equations have led to different results due to different approximations employed [1] - [7]. In the framework of dipole formulation the problem has also been studied in Ref. [8]. Since the cumulant moments $K_{q}$, even for small $q$, are very sensitive to the approximations made [6],[7], it is important to do the calculations as precisely as possible, both in the treatment of the kernels and in the handling of the coupling constant. In [2] we did the exact calculation in the case of fixed coupling for the first moment. In this paper we shall extend the same treatment to higher moments. It is remarkable that our results differ strongly from those obtained in double-logarithm approximation (DLA) of QCD which is insensitive to whether coupling is running or fixed [9].

Before we get into the full complication of the QCD problem, it is instructive to examine some simple cases for illustrative purposes (see also [10],[11]). There are two aspects to this work. One is to determine the dynamical predictions on the factorial moments $F_{q}$. The other is to calculate from $F_{q}$ the cumulant moments $K_{q}$. The former involves solving the QCD equations. We shall do that, however, after showing what the latter involves, assuming some standard forms for $P_{n}$ commonly considered in phenomenology. 


\section{Multiplicity moments}

To illustrate the properties of various moments, let us consider some simple examples based on (a) fixed multiplicity distribution (FMD), (b) Poisson distribution (PD), and (c) negative binomial distribution (NBD).

The generating function $G(z)$ for the multiplicity distribution $P_{n}$ is defined by

$$
G(z)=\sum_{n=0}^{\infty}(1+z)^{n} P_{n}
$$

so that the inverse is

$$
P_{n}=\left.\frac{1}{n !} \frac{d^{n} G(z)}{d z^{n}}\right|_{z=-1} .
$$

The normalized factorial moments $F_{q}$ are

$$
F_{q}=\frac{\sum_{n=0}^{\infty} n(n-1) \ldots(n-q+1) P_{n}}{\langle n\rangle^{q}}=\left.\frac{1}{\langle n\rangle^{q}} \frac{d^{q} G(z)}{d z^{q}}\right|_{z=0},
$$

and the normalized cumulant moments $K_{q}$ are

$$
K_{q}=\left.\frac{1}{\langle n\rangle^{q}} \frac{d^{q} \ln G(z)}{d z^{q}}\right|_{z=0} .
$$

They are related to $F_{q}$ by the formula (see e.g. [12])

$$
F_{q}=\sum_{l=0}^{q-1} C_{q-1}^{l} K_{q-l} F_{l}
$$

where $C_{q}^{l}=q ! / l !(q-l) !$.

For completeness, we give a quick derivation of (5) here in the form that we shall need later. The generating function $G(z)$ is related to $F_{q}$ and $K_{q}$ by

$$
\begin{aligned}
& G(z)=\sum_{q=0}^{\infty} z^{q} A_{q}, \quad A_{q}=\langle n\rangle^{q} F_{q} / q ! \\
& \ln G(z)=\sum_{q=1}^{\infty} z^{q} B_{q}, \quad B_{q}=\langle n\rangle^{q} K_{q} / q !
\end{aligned}
$$

From the identity $d G / d z=G(d \ln G / d z)$ we immediately get $A_{1}=B_{1}$ and, for $q \geq 2$,

$$
A_{q}=B_{q}+\sum_{l=1}^{q-1} \frac{l}{q} A_{l} B_{q-l}
$$


which is equivalent to (5). For a few lower integer values of $q$ the relation (5) gives

$$
\begin{aligned}
& F_{2}=1+K_{2}, \\
& F_{3}=1+3 K_{2}+K_{3} .
\end{aligned}
$$

Although $K_{q}$ can be determined from $F_{q}$, for the simple cases to be considered they can be calculated directly from $G(z)$. We shall find their ratio also to be of interest:

$$
H_{q}=K_{q} / F_{q} .
$$

In some cases this ratio reveals the $q$ dependence better than $K_{q}$ itself [6],[10].

(a) Fixed multiplicity distribution (FMD).

For fixed $n_{0}$, we have $P_{n}=\delta_{n n_{0}}$, so $G(z)=(1+z)^{n_{0}}$. Then $\langle n\rangle=n_{0}$ and we get

$$
\begin{aligned}
& F_{q}=\frac{n_{0} !}{n_{0}^{q}\left(n_{0}-q\right) !}, \quad 1<q \leq n_{0}, \\
& K_{q}=\left(-n_{0}\right)^{1-q}(q-1) ! .
\end{aligned}
$$

Note that $K_{q}$ oscillates in sign, while its magnitude first decreases until $q=n_{0}$; for $q>n_{0}+1,\left|K_{q}\right|$ increases monotonically. This is a very common pattern for $K_{q}$, which we shall encounter again below. $F_{q}$, however, decreases monotonically with $q$ until $n_{0}$; this decrease is not common, being specifically a property of FMD.

Since $F_{q}=0$ for $q>n_{0}, H_{q}$ can be calculated up to $q=n_{0}$. In Fig.1 we show $F_{q}, K_{q}, H_{q}$ for $n_{0}=10$, and in the insets we show $\ln \left|K_{q}\right|$ and $\ln \left|H_{q}\right|$ for integer values of $q$.

(b) Poisson distribution (PD).

For PD we have

$$
P_{n}=\frac{\langle n\rangle^{n}}{n !} e^{-\langle n\rangle}, \quad G(z)=e^{\langle n\rangle z} .
$$

Thus it follows from (3) and (4) that

$$
F_{q}=1, \quad K_{q}=\delta_{q 1}
$$


and $H_{q}$ is not any more interesting.

(c) Negative binomial distribution (NBD).

For NBD the generating function is

$$
G(z)=\left(1-\frac{z\langle n\rangle}{k}\right)^{-k}
$$

so one gets for the multiplicity distribution

$$
P_{n}=\frac{\Gamma(n+k)}{\Gamma(n+1) \Gamma(k)}\left(\frac{\langle n\rangle}{k}\right)^{n}\left(1+\frac{\langle n\rangle}{k}\right)^{-n-k} .
$$

The Bose-Einstein distribution is a special case of NBD with $k=1$. In (16) and (17) $k$ need not be an integer. The corresponding moments are

$$
\begin{aligned}
F_{q} & =\frac{\Gamma(k+q)}{\Gamma(k+1) k^{q-1}}, \\
K_{q} & =\frac{\Gamma(q)}{k^{q-1}}, \\
H_{q} & =\frac{\Gamma(q) \Gamma(k+1)}{\Gamma(k+q)}=k B(q, k),
\end{aligned}
$$

where $B(q, k)$ is the Euler beta function. Here $F_{q}$ rises very rapidly with $q$, faster than exponential. Unlike the case of FMD, $K_{q}$ does not oscillate. It starts decreasing very fast, reaches a minimum at $q \approx k$, and then increases for $q>k$. $H_{q}$, however, decreases monotonically.

In Fig. 2 we show their behaviors by plotting $\ln F_{q}, \ln K_{q}$ and $\ln H_{q}$ vs $q$ for $k=5$ and 10. Since $P_{n}$ is narrower at higher $k$, the slower rise of $F_{q}$ for $k=10$ compared to that for $k=5$ is expected. The dependence of $K_{q}$ on $k$ is more pronounced than that of $H_{q}$. These properties are more characteristic of NBD than general.

It should be noted that FMD and NBD represent two opposite types of distributions, resulting in $F_{q}^{F M D}$ decreasing and $F_{q}^{N B D}$ increasing with $q$. Remarkably, $\ln \left|K_{q}^{F M D}\right|$ and $\ln K_{q}^{N B D}$ are very similar, despite the notable difference that $K_{q}^{F M D}$ oscillates, while $K_{q}^{N B D}$ does not. It is in comparing $\ln \left|H_{q}^{F M D}\right|$ with $\ln H_{q}^{N B D}$ that we see a distinct difference. These very contrasting $q$ dependencies will serve as our guidelines in appreciating the results from QCD calculations, which we now turn to. 


\section{$3 \quad$ Fixed-coupling QCD}

Before getting involved with the technical details of solving exactly the problem of fixed-coupling QCD, we start from the beginning with the general relations and discuss previous works that treat the problem with different approximations. As usual with all theoretical investigations, we consider only the evolution of partons and neglect all complications that arise in hadronization.

We treat the problem with both quarks and gluons taken into account in jets of either type. Let $P_{n}^{Q}(y)$ and $P_{n}^{G}(y)$ denote the parton multiplicity distributions in quark and gluon jets, respectively, where

$$
y=\ln \left(Q / Q_{0}\right)
$$

$Q$ being the virtuality of the jet, and $Q_{0}$ some fixed scale. The corresponding generating functions are

$$
G_{G, Q}(y, z)=\sum_{n=0}^{\infty}(1+z)^{n} P_{n}^{G, Q}(y)
$$

They can be expressed in terms of the normalized factorial moments as follows

$$
G_{G}=\sum_{q=0}^{\infty} \frac{z^{q}}{q !}\left\langle n_{G}\right\rangle^{q} F_{q}, \quad G_{Q}=\sum_{q=0}^{\infty} \frac{z^{q}}{q !}\left\langle n_{Q}\right\rangle^{q} \Phi_{q},
$$

where $\left\langle n_{G}\right\rangle$ and $\left\langle n_{Q}\right\rangle$ are the average multiplicities in the gluon and quark jets, respectively.

With the $z$ dependence suppressed (the following equations being diagonal in $z$ ), the generating functions satisfy the coupled integral equations [1]

$$
\begin{array}{r}
G_{G}^{\prime}(y)=\int_{0}^{1} d x K_{G}^{G}(x) \gamma_{0}^{2}\left[G_{G}(y+\ln x) G_{G}(y+\ln (1-x))-G_{G}(y)\right] \\
+n_{f} \int_{0}^{1} d x K_{G}^{Q}(x) \gamma_{0}^{2}\left[G_{Q}(y+\ln x) G_{Q}(y+\ln (1-x))-G_{G}(y)\right], \\
G_{Q}^{\prime}(y)=\int_{0}^{1} d x K_{Q}^{G}(x) \gamma_{0}^{2}\left[G_{G}(y+\ln x) G_{Q}(y+\ln (1-x))-G_{Q}(y)\right],
\end{array}
$$

where $G^{\prime}(y)=d G / d y, \gamma_{0}^{2}=6 \alpha_{S} / \pi, \alpha_{S}$ is QCD coupling, $n_{f}$ is the number of active flavors, and the kernels are

$$
K_{G}^{G}(x)=\frac{1}{x}-(1-x)[2-x(1-x)]
$$




$$
\begin{aligned}
& K_{G}^{Q}(x)=\frac{1}{4 N_{c}}\left[x^{2}+(1-x)^{2}\right], \\
& K_{Q}^{G}(x)=\frac{C_{F}}{N_{c}}\left(\frac{1}{x}-1+\frac{x}{2}\right),
\end{aligned}
$$

where $N_{c}=3$ is the number of colors and $C_{F}=4 / 3$.

For gluodynamics in the absence of quarks, eq.(24) survives with $n_{f}=0$. Its solution has been found in various approximations [4]-[6] depending on assumptions about the importance of the non-singular terms in kernel (26), of the running property of QCD coupling $\alpha_{S}$ or of the $x$-dependence of generating functions (which takes into account energy conservation when two gluons recombine into one). Their application to the full QCD case of eqs.(24) and (25) is straightforward (see e.g. [4],[11]) and shows that quarks do not change the qualitative features of the solution.

In this section we show that the set of equations (24), (25) can be solved exactly for fixed coupling QCD, i.e. when $\gamma_{0}$ is held constant. No other assumptions are involved. The consequences of (24) and (25) on $\left\langle n_{G}\right\rangle$ and $\left\langle n_{Q}\right\rangle$, which correspond to considering only $q=0$ and 1 in the expansions (23), have already been examined for fixed coupling QCD in Ref.[2]. We now consider the $q \geq 2$ terms. We write $\left\langle n_{G, Q}\right\rangle$ in the form

$$
\left\langle n_{G}(y)\right\rangle=e^{\gamma y}, \quad\left\langle n_{Q}(y)\right\rangle=e^{\gamma y} / r,
$$

and treat $\gamma$ as constant, an assumption which we later show to be consistent with (24) and (25); $r$ is the ratio

$$
r=\left\langle n_{G}\right\rangle /\left\langle n_{Q}\right\rangle
$$

We further assume that $F_{q}$ and $\Phi_{q}$ are independent of $y$ (again to be checked $a$ posteriori) so that (29) exhibits all the $y$ dependences in (23) explicitly.

We substitute (23) into (24) and (25), and consider only $q \geq 2$, since $q=0$ and 1 terms are decoupled from $q \geq 2$ sector when the integral equations are evaluated to $O(z)$ accuracy, resulting in self-consistency constraints within the $q<2$ sector [2]. The quadratic terms in the integrands involve displaced arguments. For example, for $G(y+\ln x)$ we have from (29)

$$
\langle n(y+\ln x)\rangle=x^{\gamma}\langle n(y)\rangle
$$


which can be used to give

$$
\begin{gathered}
G_{G}(y+\ln x) G_{G}(y+\ln (1-x))= \\
\sum_{l, m} \frac{z^{l+m}}{l ! m !} x^{l \gamma}(1-x)^{m \gamma}\left\langle n_{G}(y)\right\rangle^{l+m} F_{l} F_{m} .
\end{gathered}
$$

Excluding $q=0,1$ terms and setting $z=1$, we get from (32)

$$
\sum_{q=2}^{\infty}\left\langle n_{G}(y)\right\rangle^{q}\left\{\left[x^{g \gamma}+(1-x)^{q \gamma}\right] f_{q}+\sum_{l=1}^{q-1} x^{l \gamma}(1-x)^{(q-l) \gamma} f_{l} f_{q-l}\right\},
$$

where

$$
f_{q}=\frac{1}{q !} F_{q}, \quad \quad \phi_{q}=\frac{1}{q !} \Phi_{q} ;
$$

the latter will be needed shortly, since the other quadratic terms in (24) and (25) can be treated similarly. The linear terms involve derivatives and integrals, which can easily be expressed in terms of the moments. Hence, we obtain

$$
\begin{aligned}
& \left(\frac{q \gamma}{\gamma_{0}^{2}}-M_{q}^{G}+n_{f} N_{0,0}^{Q}\right) f_{q}-\frac{n_{f}}{r^{q}} M_{q}^{Q} \phi_{q}=\sum_{l=1}^{q-1}\left[N_{q, l}^{G} f_{l} f_{q-l}+\frac{n_{f}}{r^{q}} N_{q, l}^{Q} \phi_{l} \phi_{q-l}\right] \\
& \left(\frac{q \gamma}{\gamma_{0}^{2}}+L_{0}-L_{q, q}\right) \phi_{q}-L_{q} r^{q} f_{q}=\sum_{l=1}^{q-1} r^{q-l} L_{q, l} \phi_{l} f_{q-l}
\end{aligned}
$$

where

$$
\begin{aligned}
M_{q}^{G} & =\int_{0}^{1} d x K_{G}^{G}(x)\left[x^{q \gamma}+(1-x)^{q \gamma}-1\right] \\
M_{q}^{Q} & =\int_{0}^{1} d x K_{G}^{Q}(x)\left[x^{q \gamma}+(1-x)^{q \gamma}\right] \\
N_{q, l}^{G} & =\int_{0}^{1} d x K_{G}^{G}(x) x^{l \gamma}(1-x)^{(q-l) \gamma}, \\
N_{q, l}^{Q} & =\int_{0}^{1} d x K_{G}^{Q}(x) x^{l \gamma}(1-x)^{(q-l) \gamma}, \\
L_{q} & =\int_{0}^{1} d x K_{Q}^{G}(x) x^{q \gamma}, \\
L_{q, l} & =\int_{0}^{1} d x K_{Q}^{G}(x) x^{(q-l) \gamma}(1-x)^{l \gamma} .
\end{aligned}
$$


Upon using the kernels in (26) - (28), we have

$$
\begin{aligned}
M_{q}^{G}= & \psi(1)-\psi(q \gamma+1)+B(q \gamma, 1)-2 B(q \gamma+1,2)-2 B(q \gamma+2,1) \\
& +B(q \gamma+2,3)+B(q \gamma+3,2)+11 / 12 \\
M_{q}^{Q}= & \frac{1}{2 N_{c}}[B(q \gamma+3,1)+B(q \gamma+1,3)] \\
N_{q, l}^{G}= & B(l \gamma,(q-l) \gamma+1)-2 B(l \gamma+1,(q-l) \gamma+2) \\
& +B(l \gamma+2,(q-l) \gamma+3) \\
N_{q, l}^{Q}= & \frac{1}{4 N_{c}}[B(l \gamma+3,(q-l) \gamma+1)+B(l \gamma+1,(q-l) \gamma+3)] \\
L_{q}= & \frac{C_{F}}{N_{c}}\left[B(q \gamma, 1)-B(q \gamma+1,1)+\frac{1}{2} B(q \gamma+2,1)\right] \\
& \frac{C_{F}}{N_{c}}[B((q-l) \gamma, l \gamma+1)-B((q-l) \gamma+1, l \gamma+1) \\
& \left.+\frac{1}{2} B((q-l) \gamma+2, l \gamma+1)\right] .
\end{aligned}
$$

Given $\gamma$, these quantities are precisely known. Thus our problem has been reduced to solving $2(q-1)$ simultaneous, nonlinear algebraic equations (35) and (36).

Let us simplify the appearance of (35) and (36) by first defining

$$
\hat{\phi}_{q}=\phi_{q} / r^{q} \text {. }
$$

Next, define

$$
\begin{aligned}
& S_{q}(f, \hat{\phi})=\sum_{l=1}^{q-1}\left[N_{q, l}^{G} f_{l} f_{q-l}+n_{f} N_{q, l}^{Q} \hat{\phi}_{l} \hat{\phi}_{q-l}\right], \\
& T_{q}(f, \hat{\phi})=\sum_{l=1}^{q-1} L_{q, l} \hat{\phi}_{l} f_{q-l} .
\end{aligned}
$$

Then (35) and (36) can be reexpressed in the form

$$
\begin{aligned}
& f_{q}=\left[a_{q} S_{q}(f, \hat{\phi})+b_{q} T_{q}(f, \hat{\phi})\right] / D_{q}, \\
& \hat{\phi}_{q}=\left[c_{q} S_{q}(f, \hat{\phi})+d_{q} T_{q}(f, \hat{\phi})\right] / D_{q},
\end{aligned}
$$


where

$$
\begin{aligned}
& a_{q}=\frac{q \gamma}{\gamma_{0}^{2}}+L_{0}-L_{q, q}, \\
& b_{q}=n_{f} M_{q}^{Q}, \\
& c_{q}=L_{q}, \\
& d_{q}=\frac{q \gamma}{\gamma_{0}^{2}}-M_{q}^{G}+n_{f} N_{0,0}^{Q}, \\
& D_{q}=a_{q} d_{q}-b_{q} c_{q} .
\end{aligned}
$$

Since $S_{q}$ and $T_{q}$ involve $f_{l}$ and $\hat{\phi}_{l}$ for $l=1, \ldots, q-1$, the method of solving these equations recursively is now clear. From known moments up to rank $q-1$, evaluate $S_{q}$ and $T_{q}$, which determine $f_{q}$ and $\hat{\phi}_{q}$ by (52) and (53). The process is then repeated to the next rank.

The initial condition at $q=1$ is trivial:

$$
F_{1}=f_{1}=\Phi_{1}=\phi_{1}=1
$$

Because of that the $q \geq 2$ sector is decoupled from the $q<2$ sector except for the link through the ratio $r$ of average multiplicities of partons in gluon and quark jets, defined in $(30)$, which determines

$$
\hat{\phi}_{1}=1 / r \text {. }
$$

The value of $r$ has been calculated in Ref. [2] in the fixed coupling approximation. The result is insensitive to the number of active flavors $n_{f}$. For the range of $\gamma_{0}$ of interest, $0.48<\gamma_{0}<0.6$, we have

$$
r=1.84 \pm 0.02 \text {. }
$$

Its differences from the asymptotic QCD value $9 / 4$ and from the experimental value for hadrons $r_{\text {exp }} \approx 1.3$ are explained in Ref. [2] as being phenomenologically satisfactory. We shall proceed with our calculation in the $q \geq 2$ sector using (59) - (61) for the $q=1$ boundary. 
For every fixed $\gamma_{0}$ we shall also need $\gamma$ to determine $a_{q}, \ldots, d_{q}$ in $(54)-(57)$ as well as $S_{q}$ and $T_{q}$ in (50), (51). The dependence of $\gamma$ on $\gamma_{0}$ has also been calculated in Ref. [2]. The result can be well fitted by the formula

$$
\gamma=0.077+0.62 \gamma_{0}
$$

for the range of $\gamma_{0}$ of interest. It will be used in our calculation below.

It should be noted that the system of equations (50) - (53) has no explicit dependence on $y$. The assumption that the only $y$ dependence is in $\left\langle n_{G, Q}(y)\right\rangle$, given in (29), with constant $r$ has led to a transformation of the differentiointegral equations (24) and (25) to a set of algebraic equations (50)-(53), which are consistent with all quantities being independent of $y$. In particular, it is self-consistent to treat the moments $f_{q}$ and $\hat{\phi}_{g}$ as $y$ independent in the fixed coupling approximation. Since $r$ is constant for fixed $\alpha_{S}$, it means that $\phi_{q}$ is also $y$ independent. Thus all factorial moments are energy independent. We shall call this property $F$-scaling. Asymptotically it coincides with KNO-scaling which implies the scaling of ordinary moments. Let us stress that $F$-scaling is precise for fixed-coupling QCD.

In [2] we have shown that even if $\alpha_{S}$ is allowed to run, the dependence of $\gamma$ on $Q$ is still very mild to justify our treatment that all quantities in (50)-(58) are independent of $y$. This is the a posteriori justification we needed to carry out our reduction of the original equations on $G_{G}(y, z)$ and $G_{Q}(y, z)$.

The results of our calculation from (50) - (62), when expressed in terms of $F_{q}$ and $\Phi_{q}$, are shown in Fig.3(a) for $\gamma_{0}=0.48$ and $n_{f}=5$. Evidently, they increase rapidly with $q$, more so for $\Phi_{q}$ than $F_{q}$. Since these are normalized factorial moments (see (3) for $F_{q}$, and similar definition for $\Phi_{q}$ ), they imply that the multiplicity distribution for the quark jet $P_{n}^{Q}$ is wider than that for the gluon jet $P_{n}^{G}$, although $\left\langle n_{Q}\right\rangle<\left\langle n_{G}\right\rangle$. These results are very insensitive to the value of $n_{f}$.

Note that by comparing Fig.3(a) with Fig.1(a) and 2(a) the QCD results are clearly of the NBD type rather than the FMD. In fact, $F_{q}$ in Fig.3(a) can be approximated by $F_{q}^{N B D}$ with $k=5$.

While the characterization of $F_{q}$ by the NBD parameter $k$ is convenient, we now show how inadequate the NBD is as an approximation. Recall from Fig.2(b) that the cumulant moment $K_{q}$ for NBD decreases monotonically to $q \approx k$ and then increases. This behavior will be shown below to be badly violated for quark 
and gluon jets, thereby revealing the sensitivity of $K_{q}$ and $H_{q}$ on the detailed properties of $F_{q}$, as has been noted before [6], [10].

If we define

$$
k_{q}=K_{q} / q !
$$

and use $f_{q}$ as defined in (34), then we get from (8)

$$
k_{q}=f_{q}-\sum_{l=1}^{q-1} \frac{l}{q} f_{l} k_{q-l}
$$

with $f_{1}=k_{1}=1$. Thus the knowledge of $f_{q}$ for all $q$ enables us to calculate all $k_{q}$. Similarly, for the quark jet, we have analogous moments $\phi_{q}=\Phi_{q} / q$ ! and the cumulant counterparts $\Psi_{q}$ and $\psi_{q}$, playing the roles of $K_{q}$ and $k_{q}$, respectively, and satisfying

$$
\psi_{q}=\phi_{q}-\sum_{l=1}^{q-1} \frac{l}{q} \phi_{l} \psi_{q-l}
$$

The $H$ moments for the gluon and quark jets are then

$$
\begin{aligned}
& H_{q}=K_{q} / F_{q}=k_{q} / f_{q}, \\
& \eta_{q}=\Psi_{q} / \Phi_{q}=\psi_{q} / \phi_{q} .
\end{aligned}
$$

Since we have already determined $f_{q}$ and $\phi_{q}$ for all $q$ of interest, we can therefore calculate $K_{q}, H_{g}, \Psi_{q}$ and $\eta_{q}$ using (64) - (67). The results are shown in Figs.3(b), 4 and 5.

The distinctive feature of the behavior of $H_{q}$ is clearly its oscillations. It shows that the NBD fits are inappropriate. At the same time, the period of oscillations is larger than in the case of fixed multiplicity where $H_{q}$ alternates in sign at each $q$. Let us stress that the exact solution of fixed coupling QCD differs also strongly from the lowest order (DLA) approximation of QCD where the KNO function is insensitive to $\alpha_{S}$ being running or fixed. The latter can be taken either from previous results $[1],[5],[6],[10]$ dealing with running coupling or from the fixed coupling result without non-leading terms (35). In both cases, the ratio $H_{q}$ decreases monotonically as

$$
H_{q}=q^{-2}
$$


at asymptotically high energies. Transition to preasymptotic energies and to modified leading log approximation (MLLA) with running coupling provides some oscillations imposed on the above law or even changes it depending on whether the coupling $\gamma_{0}$ tends to zero limit first or the moment considered tends to infinity. It reflects the fact that the product $q \gamma_{0}$ appears as an expansion parameter that has been exploited in [5],[6]. It has been shown in [6] that at large $q$ and fixed $\gamma_{0}$ the asymptotic behavior of $H_{q}$ in DLA is proportional to $q^{-2}$, in MLLA it decreases as $q^{-1}$ and in the next-order approximation it tends to a constant. However, all of them are consequences of the approximations made and should not be considered as realistic quantitative results.

One can demonstrate how easy it is to get the oscillations of the FMD-type imposed on DLA-behavior by doing the following exercise. It is known [5] that the large $q$ behavior of $F_{q}$ in DLA is

$$
F_{q}^{D L A} \approx \frac{2 q \Gamma(q+1)}{C^{q}}
$$

where $C \approx 2.553$. If we add a preasymptotic term by replacing the factor $2 q$ by $2 q+1$ in the numerator, one gets an additional term in $H_{q}$ of the FMD-type so that it becomes

$$
H_{q}^{(n e w)}=\frac{2+(-1)^{q-1}}{q(2 q+1)}
$$

where $(-1)^{q-1}$ appears due to the added preasymptotic term.

The above example shows how sensitive $H_{q}$ is to various approximations made in solving the set of equations for generating functions. Therefore, the exact solution found by us here provides clear guide to the realistic behavior of $H_{q}$, since the only assumption made is of fixed coupling.

\section{Summary and discussion}

We have been able to get exact solution (52) and (53) of QCD equations for generating functions (24) and (25) in case of fixed coupling for any rank of the moment of parton multiplicity distribution in quark and gluon jets. It is the generalization of our previous result [2] on average multiplicity in quark and gluon jets. It shows that cumulant moments (or their ratio to factorial moments) are very sensitive to tiny details of the multiplicity distribution, as has been stated previously [6], [10] 
when studying various approximations to the equations and phenomenological distributions. Partons in quark jets are distributed wider in their multiplicity fluctuations than in gluon jets even though the average multiplicity of quark jets is lower.

The oscillating structure of the ratio of cumulant to factorial moments is typical for jets of both kinds, which is to be contrasted to the monotonic decrease in the cases of the negative binomial distribution and the double logarithm approximation of QCD. Similar oscillating structure appears also in higher-order approximate solutions of QCD equations with running coupling [7]. It has been supported by experimental data [13] at a qualitative level. However, before proceeding to quantitative comparison more work should be done on taking into account both the running property of QCD coupling and the relations to real $e^{+} e^{-}$and hadronic processes. Furthermore, the validity of the starting equations on the generating functions, that do not include, for example, the effects of four-gluon vertices, should be examined.

We have done this work on fixed-coupling QCD, not because it is more important than the running-coupling problem, but because it allows an exact solution that is not possible in the running case. When approximations are made, whether DLA or MLLA, it is difficult to assess how significant the errors are. The fact that inaccuracies show up sensitively in the $H_{q}$ moments provides the motivation for us to study exact solutions, even at the cost of fixing the coupling constant. By varying the value of $\gamma_{0}$ we have found that the effect on our result is small, i.e., the oscillating behavior of the $H_{q}$ moments is unchanged. Thus we believe that the fixed-coupling solution determined in this paper is not only definitive, but can also provide a guidepost to other future studies that improve upon the present work.

\section{Acknowledgements}

This work was supported in part by a NATO grant under reference CRG 930025 and by the U.S. Department of Energy under grant No. DE-FG0691ER40637. 


\section{References}

1. Yu.L. Dokshitzer, V.A. Khoze, A.H. Mueller and S.I. Troyan, Basics of Perturbative QCD, Ed. J. Tran Thanh Van, Editions Frontieres, 1991.

2. I.M. Dremin and R.C. Hwa, Preprint OITS 525, FIAN TD-26, November 1993.

3. J.B. Gaffney and A.H. Mueller, Nucl. Phys. B250 (1985) 109.

4. E.D. Malaza and B.R. Webber, Nucl. Phys. B267 (1986) 702.

5. Yu.L. Dokshitzer, Phys. Lett. B305 (1993) 295.

6. I.M. Dremin, Phys. Lett. B313 (1993) 209.

7. I.M. Dremin and V.A. Nechitailo, JETP Lett. 52 (1993) 945; Preprint OITS 520, FIAN TD-23, September 1993.

8. M. Olsson and G. Gustafson, Nucl. Phys. B406 (1993) 293.

9. A. Bassetto, M. Ciafaloni and G. Marchesini, Nucl. Phys. B163 (1980) 477; Phys. Rep. 100 (1983) 202.

10. I.M. Dremin, Mod. Phys. Lett. A8 (1993) 2747.

11. I.M. Dremin, B.B. Levtchenko and V.A. Nechitailo, Sov. J. Nucl. Phys. ( to be published); Preprint OITS 524, FIAN TD-25, November 1993.

12. E.A. DeWolf, I.M. Dremin and W. Kittel, Usp. Fiz. Nauk. 163 (1993) 3; Phys. Rep. (to be published).

13. G. Gianini, in Proc. of "Multiparticle Dynamics-93", Aspen, 1993 (to be published by World Scientific, Singapore) 


\section{Figure Captions}

Fig. 1. Moments of fixed multiplicity distribution for $n_{0}=10$. (a) $F_{q}$, (b) $K_{q}, \ln \left|K_{q}\right|$, and (c) $H_{q}, \ln \left|H_{q}\right|$.

Fig. 2. Moments of the negative binomial distribution for $k=5$ and 10. (a) $\ln F_{q}$, (b) $\ln K_{q}$, and (c) $\ln H_{q}$.

Fig. 3. Moments of multiplicity distribution in fixed-coupling QCD for $\gamma_{0}=0.48, n_{f}=5$. (a) $\ln F_{q}, \ln \Phi_{q}$ and (b) $\ln \left|K_{q}\right|, \ln \left|\Psi_{q}\right|$.

Fig.4. $\quad H_{q}$ moments of gluon-jet distribution in fixed-coupling QCD for $\gamma_{0}=0.48, n_{f}=5$. (a) $\mathrm{H}_{q}$ and (b) $\ln \left|H_{q}\right|$.

Fig. 5. $\quad \eta_{q}$ moments of quark-jet distribution in fixed-coupling QCD for $\gamma_{0}=0.48, n_{f}=5 . \quad$ (a) $\eta_{q}$ and (b) $\ln \left|\eta_{q}\right|$. 

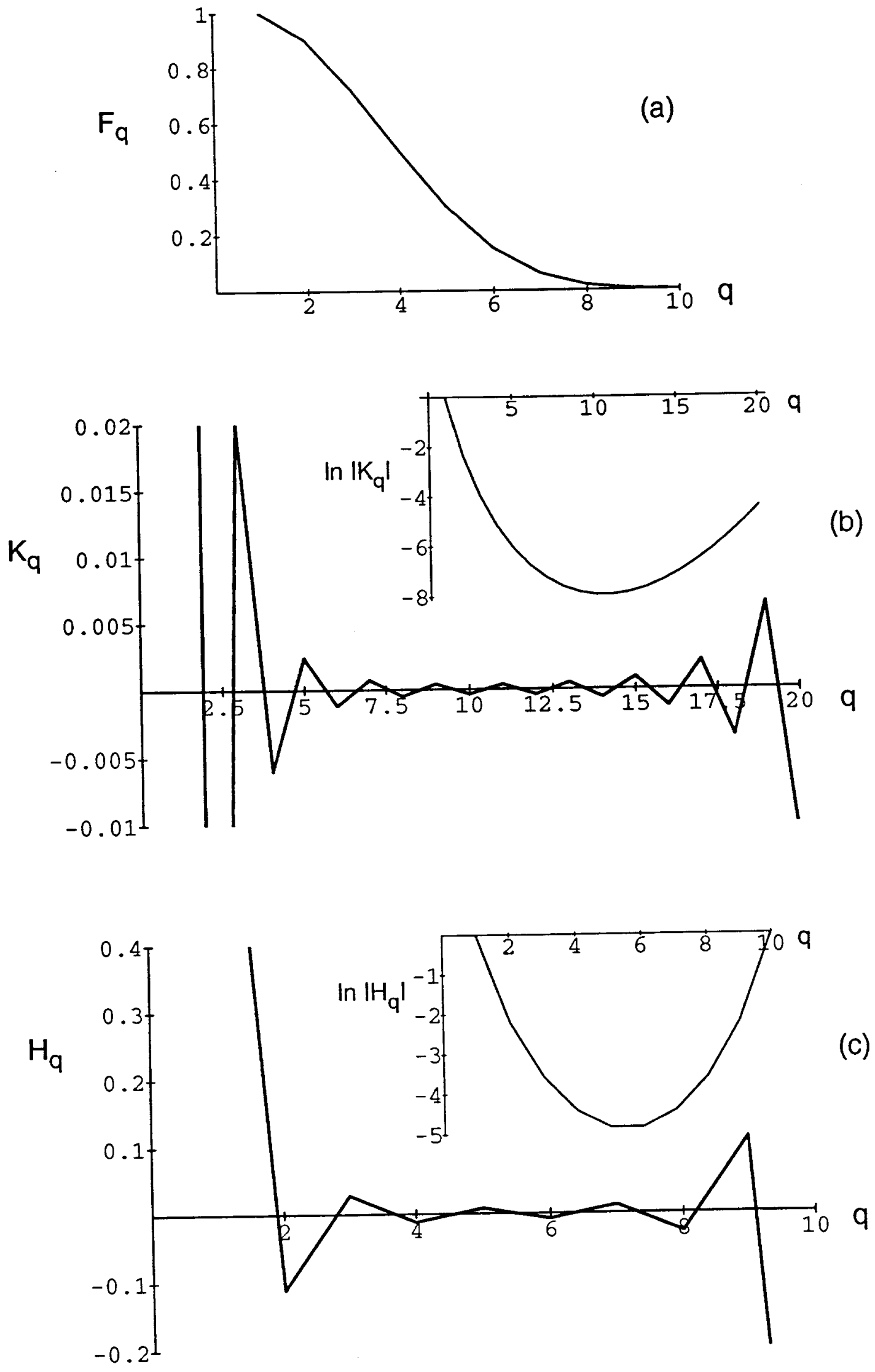

Fig. 1 

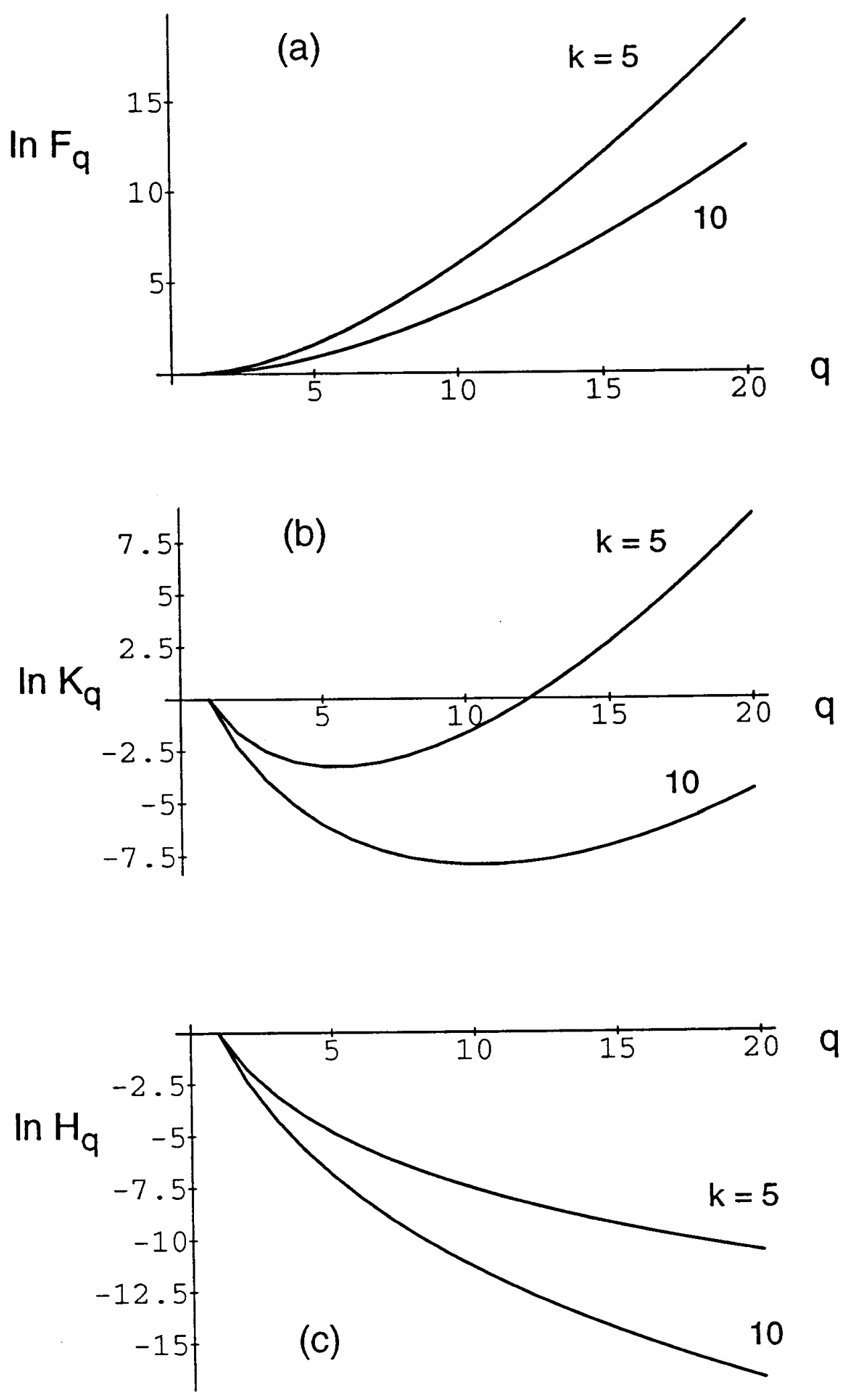

Fig. 2 

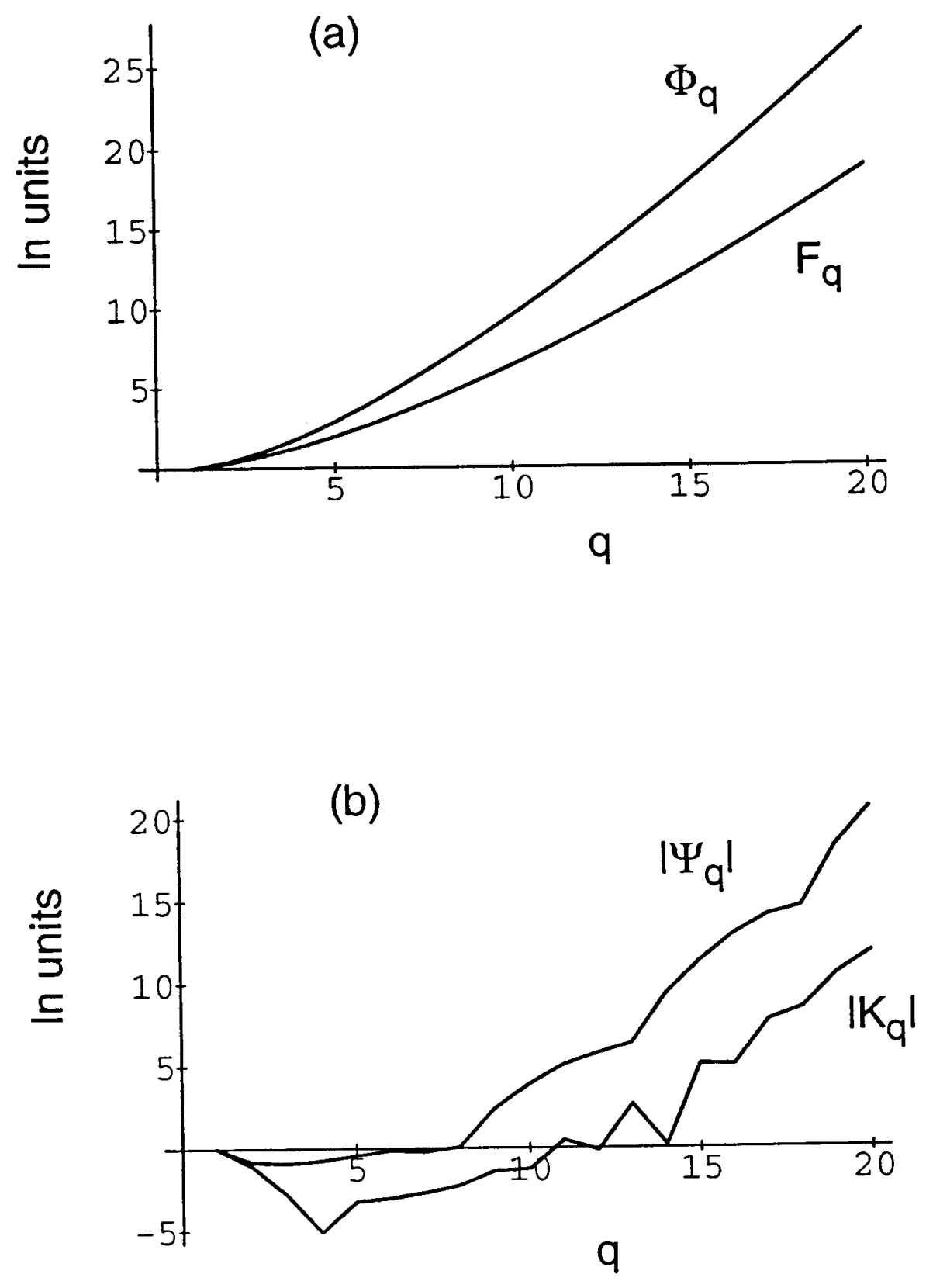

Fig. 3 

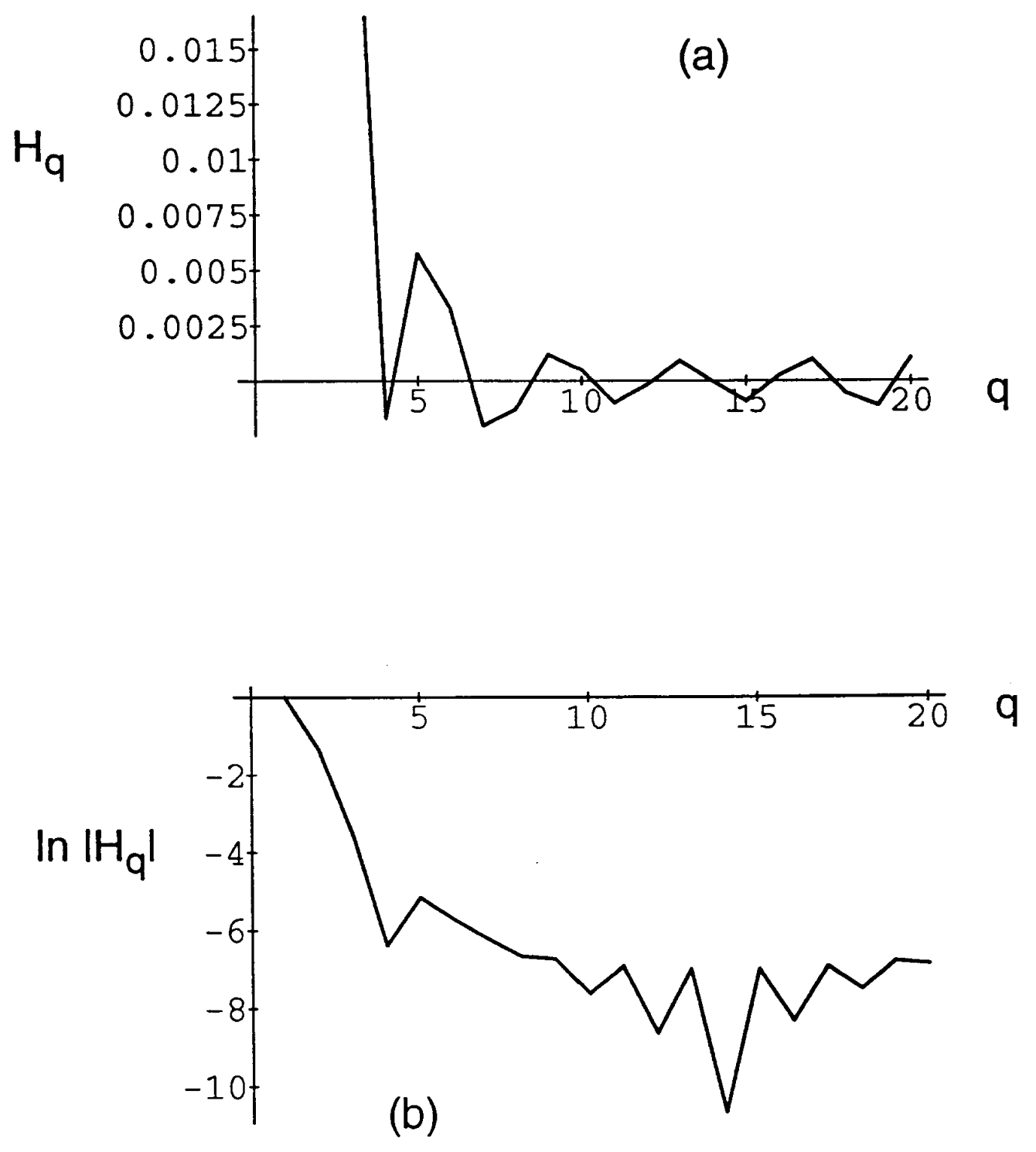

Fig. 4 

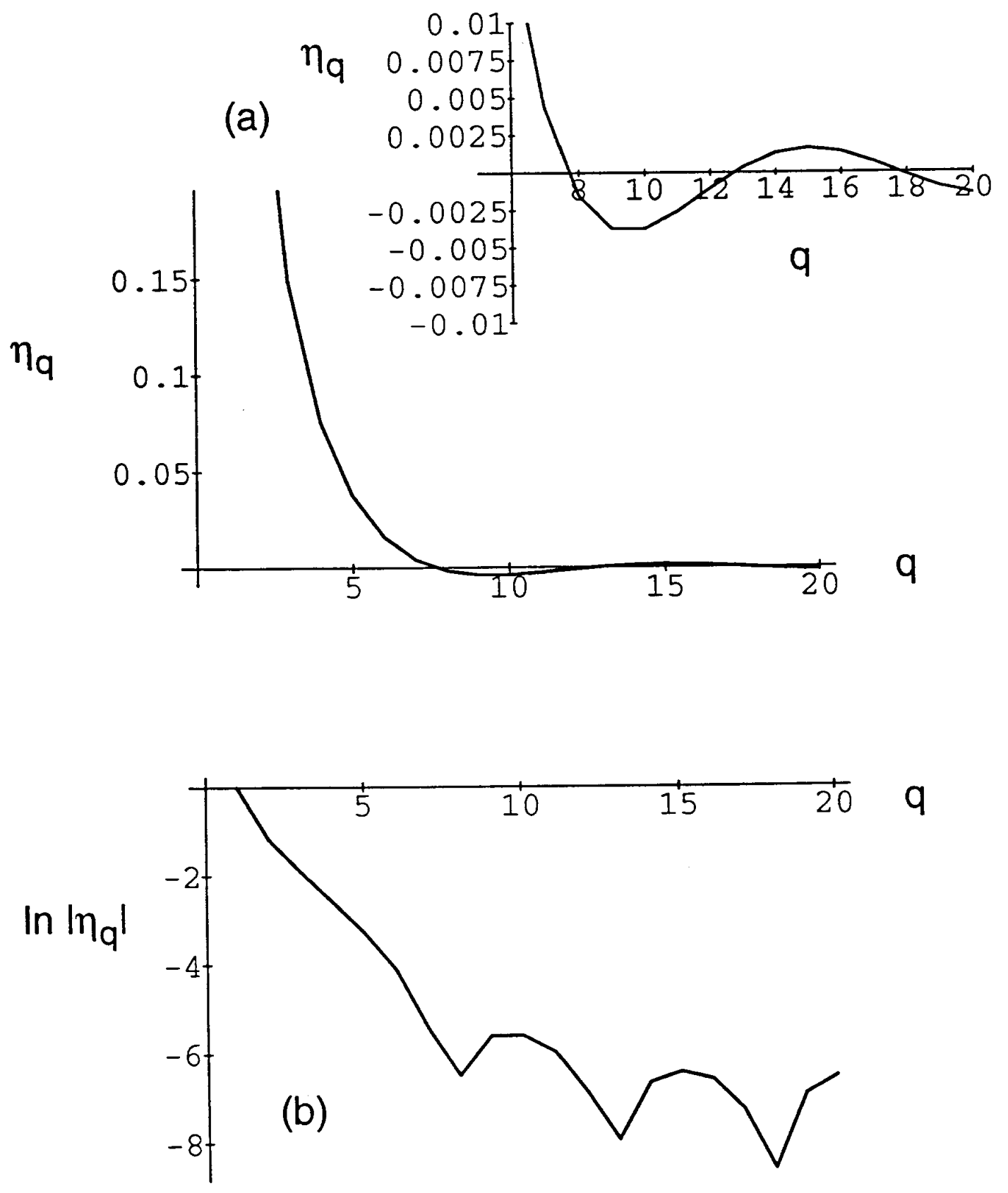

Fig. 5 